\title{
Gap Sensitivity Reveals Universal Behaviors in Optimized Photonic Crystal and Disordered Networks
}

\author{
Michael A. Klatt,${ }^{1,2, *}$ Paul J. Steinhardt,${ }^{1, \dagger}$ and Salvatore Torquato ${ }^{1,3, \ddagger}$ \\ ${ }^{1}$ Department of Physics, Princeton University, Princeton, New Jersey 08544, USA \\ ${ }^{2}$ Institut für Theoretische Physik, University of Erlangen-Nürnberg, Staudtstr. 7, 91058 Erlangen, Germany \\ ${ }^{3}$ Department of Chemistry, Princeton Institute for the Science and Technology of Materials, \\ and Program in Applied and Computational Mathematics, \\ Princeton University, Princeton, New Jersey 08544, USA
}

(Dated: July 16, 2021)

\begin{abstract}
Through an extensive series of high-precision numerical computations of the optimal complete photonic band gap (PBG) as a function of dielectric contrast $\alpha$ for a variety of crystal and disordered heterostructures, we reveal striking universal behaviors of the gap sensitivity $\mathcal{S}(\alpha) \equiv d \Delta(\alpha) / d \alpha$, the first derivative of the optimal gap-to-midgap ratio $\Delta(\alpha)$. In particular, for all our crystal networks, $\mathcal{S}(\alpha)$ takes a universal form that is well approximated by the analytic formula for a one-dimensional quarter-wave stack, $\mathcal{S}_{\mathrm{QWS}}(\alpha)$. Even more surprisingly, the values of $\mathcal{S}(\alpha)$ for our disordered networks converge to $\mathcal{S}_{\mathrm{QWS}}(\alpha)$ for sufficiently large $\alpha$. A deeper understanding of the simplicity of this universal behavior may provide fundamental insights about PBG formation and guidance in the design of novel photonic heterostructures.
\end{abstract}

Introduction. A complete photonic band gap (PBG) prohibits the propagation of light in all directions and for all polarizations for a substantial continuous range of frequencies $[1,2]$. PBGs can occur in heterostructures composed of two or more substances with different dielectric constants such as silicon and air; see Fig. 1. In early studies, PBGs were exclusively found in crystalline structures, such as the diamond crystal network [3]. Later it was discovered that quasicrystals without long-range periodic translational order $[4,5]$ and isotropic "disordered" solids can also exhibit complete PBGs [6-16].

In this paper, we present evidence for a subtle, unanticipated universal behavior of the maximum complete PBG as a function of dielectric contrast among network heterostructures spanning a wide variety of symmetries and topologies. For this purpose, we follow a multistage procedure that begins with identifying different "candidate classes" of networks distinguished by their nearestneighbor table properties (e.g., coordination), translational order and rotational symmetry which are known from past work to include examples with large complete
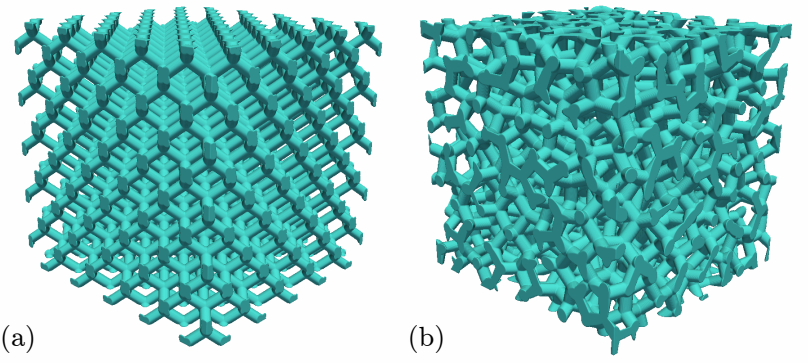

FIG. 1. Photonic networks: (a) the crystal diamond network, where cylindrical rods connect nearest neighbors of a diamond lattice, and (b) the disordered nearly hyperuniform network (NHN) model; see also video S1.
PBGs $[1-3,6,7,17]$. For each candidate class, we aim to identify the member with the largest complete PBG, which are, based on experience to date, among those that combine the highest degree of hyperuniformity [18-21] with sufficiently narrow distributions of bond lengths and angles.

Then, we optimize the microscopic properties of the selected networks. For this, we assume the networks are composed of rods and spheres with dielectric constant $\varepsilon_{2}$ embedded in a bulk that has a smaller dielectric constant $\varepsilon_{1}$. We also assume the rods have circular cross-section with radius $R$ joined at sphere-shaped vertices with the same radius $R$. For each fixed dielectric contrast ratio $\alpha:=\varepsilon_{2} / \varepsilon_{1}$, we vary $R$ to find the maximal value of the photonic band gap-to-midgap ratio $\Delta(\alpha)$; that is,

$$
\Delta(\alpha):=\max _{R \geq 0}\left\{\frac{\Delta \omega}{\omega_{m}}(R, \alpha)\right\} .
$$

We call the optimized radius $R_{\text {opt }}(\alpha)$ and the corresponding optimized volume fraction $\phi_{\text {opt }}(\alpha)$.

Since our purpose is to study cases with large PBGs, we only consider candidate classes known to have some networks satisfying this condition. In some cases, this condition is not straightforward to check. For example, disordered networks typically have many localized defect modes that break up what would otherwise be a large PBG into many smaller PBGs. As a last step of the optimization, we check whether such defects can be removed via bond switching; if so, then the candidate class is included in the study and the modified network is treated according to the same optimization procedure as described above.

Critical to our analysis are high-precision calculations of the optimal gap-to-midgap ratios, $\Delta(\alpha)$, that are unprecedented in their scope, both for the wide variety of heterostructures considered and the wide range of dielec- 
tric contrasts. Performing this computation for disordered networks with 1000 vertices is only first possible now using state-of-the-art computational techniques and computer clusters. To get reliable results for $\Delta(\alpha)$ and hence $\mathcal{S}(\alpha)$, it is essential to (i) accurately determine the optimized volume fraction $\phi_{\text {opt }}(\alpha)$; (ii) compute the stopgap along a number of different directions of propagation; (iii) use a sufficiently high spatial resolution; and (iv) use a precise plane wave expansion method to solve Maxwell's equation [22, 23].

A cursory view of plots of $\Delta(\alpha)$ as a function of $\alpha$ already suggests certain common trends across the different types of networks (despite the distinctly different functional values). What proves to be the key to revealing the universal behaviors is the gap-sensitivity, $\mathcal{S}(\alpha)$, defined as

$$
\mathcal{S}(\alpha):=\frac{d \Delta(\alpha)}{d \alpha} .
$$

As we show below, for all our optimized threedimensional crystal networks, $\mathcal{S}(\alpha)$ is well approximated for all $\alpha$ by

$$
\mathcal{S}_{\mathrm{QWS}}(\alpha):=\frac{2}{\pi} \frac{1}{\alpha^{3 / 4}(1+\sqrt{\alpha})},
$$

where $\mathcal{S}_{\mathrm{QWS}}(\alpha)$ is the precise analytic result for the gapsensitivity of a one-dimensional quarter-wave stack composed of alternating layers with dielectric constants $\varepsilon_{1}$ and $\varepsilon_{2}$ and each of quarter-wavelength thickness (which results in an optimized volume fraction) where the propagation of electromagnetic waves is perpendicular to the alternating dielectric layers. For a derivation of the corresponding formula for $\Delta(\alpha)$; see, e.g., Refs. [24, 25].

Perhaps even more striking is the fact that, for all our optimized disordered networks, the values of $\mathcal{S}(\alpha)$ also converge to $\mathcal{S}_{\mathrm{QWS}}(\alpha)$ at large $\alpha$. In other words, at sufficiently large $\alpha$, all our optimized crystal and disordered heterostructures - despite the differences in symmetry, topology, and long-range order - exhibit the same universal behavior: $\mathcal{S}(\alpha) \approx \mathcal{S}_{\mathrm{QWS}}(\alpha)$.

Notably, the story is different for small $\alpha=\mathcal{O}(1)$ near the critical value $\alpha_{c}$ (defined as the minimal contrast at which a complete PBG first appears). Over this range, $\mathcal{S}(\alpha)$ displays a common pattern for all our optimized disordered networks that is clearly distinct from the common pattern for crystal networks. Namely, whereas $\mathcal{S}(\alpha)$ for the crystal networks decreases monotonically even for small $\alpha, \mathcal{S}(\alpha)$ for disordered networks first increases, reaches a maximum, and then decreases, ultimately converging to $\mathcal{S}_{\mathrm{QWS}}(\alpha)$ as $\alpha$ continues to increase.

Candidate classes of networks. Among the abundant variety of known photonic crystals and disordered heterostructures, we select, for our computationally intensive optimization, candidate classes of networks that represent a broad spectrum of symmetries and coordination numbers. The diamond crystal network exhibits the largest known optimal gap-to-midgap ratio
$\Delta(\alpha)[2,3,17]$. The hexagonal-diamond network has the same topology and perfectly tetrahedral vertices but a different symmetry. It has substantial PBGs, although its optimal gap-to-midgap ratios $\Delta(\alpha)$ are distinctly smaller than those of the diamond network [26]. A better performing photonic crystal is the rod-connected network based on the Laves graph, which has the same topology as the single gyroid $[2,15,27,28]$. The diamond and Laves graphs are the only two crystal lattices in three dimensions with the "strong isotropic property" [29], i.e., a symmetry under permutations of neighboring edges. The key differences are that the diamond network is fourfold coordinated and nonchiral with a face-centered cubic symmetry and the Laves graph is trivalent and chiral with a body-centered cubic symmetry. We also study a simple cubic (SC) heterostructure, a sixfold-coordinated network consisting of rods connecting nearest neighbors in a SC lattice [30, 31].

Our isotropic disordered networks are not based on any underlying lattice. They exhibit a correlated disorder with a varying degree of both local and global order. Based on experience to date, the member in a candidate class with the largest PBG has bond length and angle distributions with a standard deviation less than $15 \%$ of the mean. For larger variations, the PBG becomes smaller and the absolute difference between $\mathcal{S}(\alpha)$ and $\mathcal{S}_{\text {QwS }}(\alpha)$ becomes larger.

One class of disordered models that we consider are continuous random networks (CRNs), i.e., idealized models for amorphous tetrahedrally coordinated solids (like amorphous silicon) $[19,32]$. The first complete PBG of three-dimensional disordered networks was found for CRNs [7]. Here, we study the PBGs of the nearly hyperuniform network (NHN) model [19]. Starting from a classical CRN, the model is carefully annealed to suppress large-scale density fluctuations. The model is thus driven towards a vanishing of density fluctuations in the infinitewavelength limit, known as hyperuniformity [18, 33]. In two dimensions, hyperuniformity has been found advantageous for opening up large complete PBGs [6].

Another disordered network that we consider is based on an alternative structural model of amorphous silicon that was simulated by a slow quench of a liquid using molecular dynamics (MD) [20]. When we connected each atom with its four nearest neighbors, we obtained a disordered photonic network, but it had defect modes that appeared within a large PBG. We, therefore, switched bonds to remove the defect modes, nearly doubling the gap size.

Finally, we introduce quantizer-based networks (QBNs). We begin from amorphous, nearly hyperuniform inherent structures of the quantizer energy starting from some random initial point configuration [21]. For more details on the quantizer energy functional; see supplemental material (SM) Sec. 1 and Refs. [21, 34-41]. Next, we construct the corresponding Delaunay tessella- 


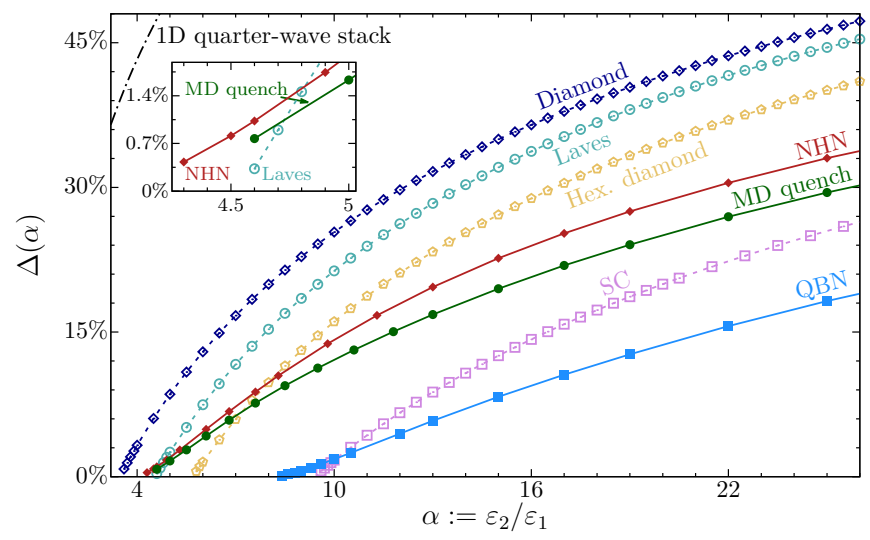

FIG. 2. The gap plot shows the optimal gap-to-midgap ratio $\Delta(\alpha)$ as a function of the dielectric contrast $\alpha$. The plot compares three-dimensional photonic crystals (open symbols) to three-dimensional disordered networks (solid symbols). The dashed-dotted line shows $\Delta_{\mathrm{QWS}}(\alpha)$ for the one-dimensional quarter-wave stack. The inset zooms in on small values of $\alpha$ where the PBGs first open up for the NHN, MD quench, and Laves networks.

tion. A QBN then connects the centroids of neighboring cells, in analogy to the tiling procedure for hyperuniform disordered solids (HUDS) in two dimensions described in Ref. [6]. In three dimensions, the tiling procedure was already applied to hard-sphere packings [9, 12, 42] and vertex models [16]. To avoid defect modes for $\alpha>13$, we removed each vertex where two triangles met by bond switching.

Our three disordered networks fulfill all of our selection criteria. In particular, the ratio of standard deviations to mean values for the bond lengths and angles are $3.6 \%$ and $7.4 \%$ for the NHN, $1.9 \%$ and $8.5 \%$ for the MD quench, and $7.4 \%$ and $10.7 \%$ for the QBN.

Protocol. All samples are constructed with periodic boundary conditions to enable band structure calculations. The PBGs are accurately determined using the plane-wave expansion method to solve the frequencydomain eigenproblem implemented in the MIT Photonic Bands (MPB) software package [22]. The plane-wave expansion is the best available method for achieving the high precision needed for the optimization and analysis presented here. It is not clear whether the same accuracy can be achieved using time-domain eigensolvers, like the finite-difference time-domain (FDTD) method, which may miss eigenfrequencies or locate spurious ones [1]. We define a complete PBG by a range of frequencies for which there are no states no matter the direction of the wave vector. In practice, we compute the stopgaps for a finite number of directions along the edges of the irreducible Brillouin zone.

For each sample and each dielectric contrast $\alpha$, we optimize the rod radius $R$ and hence the volume fraction $\phi$ of the high dielectric phase. We first determine the

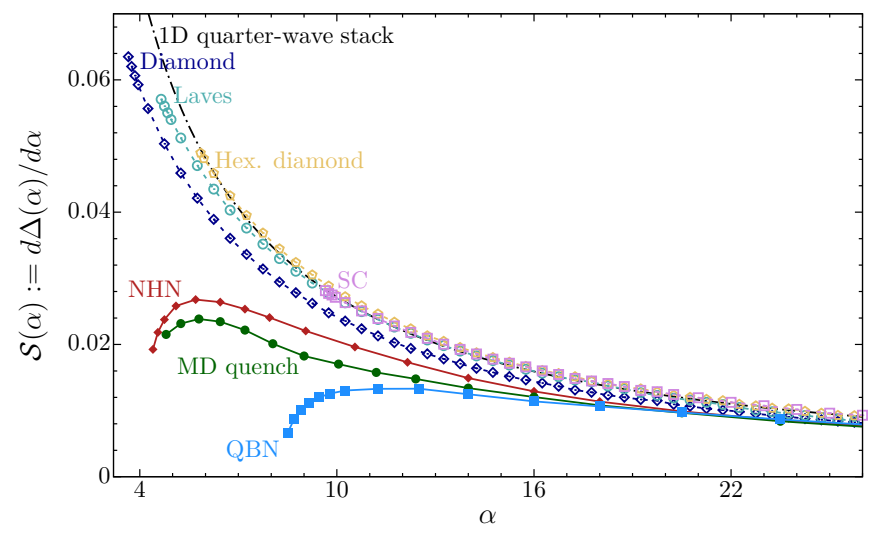

FIG. 3. The gap-sensitivity plot shows the slope $\mathcal{S}(\alpha):=$ $d \Delta(\alpha) / d \alpha$ for each of the curves in Fig. 2. For our threedimensional crystal networks, $\mathcal{S}(\alpha)$ is well approximated by $\mathcal{S}_{\mathrm{QWS}}(\alpha)$ for all $\alpha$. For our disordered networks, $\mathcal{S}(\alpha)$ has a maximum at low $\alpha=\mathcal{O}(1)$ and only converges (approximately) to $\mathcal{S}_{\mathrm{QWS}}(\alpha)$ at larger $\alpha \gg 1$.

gap-to-midgap ratios $\Delta \omega / \omega_{m}$ for several radii close to the putative optimum PBG. Next, we fit a parabola to estimate the optimized radius $R_{\text {opt }}(\alpha)$, at which we then repeat the band structure calculations. Thus, we determine the optimal gap-to-midgap ratio $\Delta(\alpha)$; see Eq. (1). We estimate $\mathcal{S}(\alpha)$, see Eq. (2), using the symmetric difference quotient.

The combined absolute error depends on the type of network and dielectric contrast and is significantly smaller at low than at high $\alpha$. Therefore, we limit our investigation to values of $\alpha \leq 26$. The error in determining $\Delta(\alpha)$ is less than $0.7 \%$ for $\alpha \leq 13$ and less than about $1.4 \%$ for $\alpha>13$. As shown in Fig. S4, the results are not sensitive to system size.

Gap plots and gap-sensitivity plots. The key results of this paper derive from Figs. 2 and 3. Figure 2 shows plots of the optimal gap-to-midgap ratio $\Delta(\alpha)$ as a function of $\alpha$ (referred to henceforth as the gap plot) for all of our crystal and disordered networks with different symmetries and topological characteristics. Figure 3 (the gapsensitivity plot) shows the slope $\mathcal{S}(\alpha):=d \Delta(\alpha) / d \alpha$ for the same networks.

Results. Photonic band structures of optimized crystal and disordered networks considered in this work are shown in Fig. S1 and video S2. The gap plot in Fig. 2 shows that the diamond network exhibits the largest PBG for all dielectric contrasts, closely followed by the Laves network. At $\alpha>10$, disordered networks have smaller PBGs than the Laves and hexagonal-diamond networks. However, $\Delta(\alpha)$ of the disordered networks increases more slowly as a function of $\alpha$ than those of the crystal networks, leading to a notable crossing of curves in the gap plot. In fact, we find a smaller critical contrast, $\alpha_{c}=4.1$, for the NHN model and MD quench than for the hexagonal-diamond network $\left(\alpha_{c}=5.8\right)$ or even the 
Laves network $\left(\alpha_{c}=4.5\right)$. Only the diamond network has a smaller critical contrast $\left(\alpha_{c}=3.5\right)$. Moreover, the gap plot shows that the NHN model has a larger optimal gap-to-midgap ratio $[\Delta(13)=19.7 \%]$ than the currently reported record for CRNs (18\%) at $\alpha=13$ [7].

Turning now to the gap-sensitivity plot in Fig. 3, we note that for all our crystal networks and for all $\alpha, \mathcal{S}(\alpha)$ is well approximated without any fit parameter by $\mathcal{S}_{\text {QwS }}(\alpha)$ from Eq. (3), the analytic result for the quarter-wave stack. Remarkably, even for our isotropic disordered networks, $\mathcal{S}(\alpha)$ converges at large $\alpha$ to $\mathcal{S}_{\mathrm{QWS}}(\alpha)$ to a good approximation. More precisely, we use "convergence" in this paper to mean $\mathcal{S}(\alpha)=(1 \pm 0.2) \mathcal{S}_{\mathrm{QWS}}(\alpha)$ for $\alpha \gtrsim 20$, corresponding to an absolute error of $2 \times 10^{-3}$, which we estimate to be the systematic error associated with the optimization protocol used to identify the member of a candidate class with the topology, bond length and angle distributions, and volume fraction that maximizes the PBG. Convergence to within this $20 \%$ uncertainty is impressive given the diverse candidate classes that have been considered: classes with highly isotropic and anisotropic PBGs; with uniform and irregular topologies; with translational order and disorder; and with onedimensional layered structure and three-dimensional network structure.

We emphasize that the multistage optimization protocol is essential to achieving this level of convergence. Without any optimization, the difference between $\mathcal{S}(\alpha)$ and $\mathcal{S}_{\mathrm{QWS}}(\alpha)$ would increase by an order of magnitude or more. Even though $\mathcal{S}(\alpha)$ is approximately the same for our three, four, and sixfold coordinated networks, we only find the universal behavior for networks with relatively uniform bond lengths and angles, as required in the first stage of our procedure. To examine the effect of variations in bond lengths and angles, we randomly perturbed the vertices of a diamond network using independent and isotropic Gaussian displacements. The strength of perturbations is controlled by the ratio $a$ of the standard deviation of the displacements to the bond length in the unperturbed network. For $a \leq 10 \%$, the perturbed diamond network fulfills our selection criterion for bond length and angles, as mentioned above, and $\mathcal{S}(\alpha)$ approximately converges to $\mathcal{S}_{\mathrm{QWS}}(\alpha)$ at large $\alpha$. However, with increasing strength of perturbations $a$, we find that the difference between $\mathcal{S}(\alpha)$ and $\mathcal{S}_{\mathrm{QWS}}(\alpha)$ increasingly grows until the PBG closes altogether when $a \approx 0.5$. We find the same behavior for a perturbed CRN [32]. The qualitative behavior of $\mathcal{S}(\alpha)$ remains the same, but $\mathcal{S}(\alpha)$ deviates from $\mathcal{S}_{\mathrm{QwS}}(\alpha)$ approximately by a constant factor that grows as $a$ increases.

The second stage of our procedure entails finding the optimized volume fraction. This step is crucial because $\phi_{\text {opt }}(\alpha)$ varies between $8.0 \%$ and $38.4 \%$ for our networks and values of $\alpha$ so that, without optimizing $\phi$, we obtain both quantitatively and qualitatively different results. If we only optimized $\phi$ to within a certain percentage of the true optimal value, we observe similar effects as for large variations in the bond lengths and angles: the difference between $\mathcal{S}(\alpha)$ and $\mathcal{S}_{\mathrm{QWS}}(\alpha)$ increases with an increasing percentage difference between $\phi$ and $\phi_{\text {opt }}(\alpha)$. Alternatively, if we fix $\phi$ independent of $\alpha$, we observe a more dramatic effect: the gap-to-midgap ratio quickly flattens for large $\alpha$ at a value well below the optimal $\Delta(\alpha)$ of that candidate class; that is, $\mathcal{S}(\alpha)$ falls rapidly to zero at large $\alpha$.

Finally, the third stage is necessary to take account of localized defect modes that break up an otherwise large PBG. These defects not only decrease $\Delta(\alpha)$ but also lead to large deviations between $\mathcal{S}(\alpha)$ and $\mathcal{S}_{\mathrm{QWS}}(\alpha)$. We observed such localized defects in the initial QBN model wherever two triangles met at one vertex. When we removed these triangle defects by bond switching, $\Delta(\alpha)$ increased significantly and $\mathcal{S}(\alpha)$ approached $\mathcal{S}_{\mathrm{QWS}}(\alpha)$ at large $\alpha$.

Although we do not have a theoretical explanation of the universal behavior of $\mathcal{S}(\alpha)$ at large $\alpha$ and the systematic differences between crystal versus disordered networks at small $\alpha$, we find an interesting correlation with the behavior of the square of the magnitude of the electric field eigenmodes $\mathbf{E}(\mathbf{r}, \alpha)$ just below and above the PBG. More precisely, we find that $\|\mathbf{E}(\mathbf{r}, \alpha)\|^{2}$ changes more at small $\alpha$ for a given change in dielectric contrast $\alpha$ than it does at large $\alpha$ and that this effect is much more pronounced for the disordered than for the crystal networks. To quantify this effect, we introduce, as a heuristic measure, the average change of $\|\mathbf{E}(\mathbf{r}, \alpha)\|^{2}$ with the dielectric contrast: $\mathcal{D}_{\mathbf{E}}(\alpha):=\int\left|\partial\|\mathbf{E}(\mathbf{r}, \alpha)\|^{2} / \partial \alpha\right| d \mathbf{r}$. Figure S2 shows that $\mathcal{D}_{\mathbf{E}}(\alpha)$ is, apart from statistical and numerical fluctuations, a decreasing function. At low $\alpha$, the changes are distinctly stronger for the disordered than the crystal networks. In contrast, at large $\alpha$, where we observe the universal behavior of $\mathcal{S}(\alpha), \mathcal{D}_{\mathbf{E}}(\alpha)$ converges within the computational uncertainty for the crystal and disordered networks.

This observation suggests the following argument: At large $\alpha$, the electric field is strongly confined by the highdielectric material, and the configuration changes only slowly with the dielectric contrast. At small $\alpha$, the electric field is generically less confined to the high-dielectric material allowing more degrees of freedom to be accessed when optimizing the PBG. The high degree of translational order in crystal systems constrains the field configuration possibilities, so the corresponding curves of $\mathcal{S}(\alpha)$ still follow Eq. (3). For disordered systems, though, there are not the same symmetry constraints, allowing more field configurations that enable the optimized PBG to decrease more slowly as $\alpha$ decreases.

We also observe that the derivative of the optimal volume fraction, $d \phi_{\text {opt }}(\alpha) / d \alpha$, approximately agrees for all our three-dimensional crystal networks at all $\alpha$ and is, in fact, well approximated by the analytic result for the one-dimensional quarter-wave stack: $d \phi_{\mathrm{QwS}}(\alpha) / d \alpha=$ 
$-1 /\left(2 \alpha^{3 / 2}+4 \alpha+2 \sqrt{\alpha}\right)$. Similarly, for our disordered networks, $d \phi_{\text {opt }}(\alpha) / d \alpha$ approximately converges to the same formula at large $\alpha$; see Fig. S3. This behavior correlates with our finding that the optimization of the volume fraction is an essential part of our multistage procedure to reveal the universal behavior of $\mathcal{S}(\alpha)$.

Conclusions and Outlook. For all our candidate classes, including ordered and disordered varieties with different symmetries and topologies, $\mathcal{S}(\alpha)$ of our optimized three-dimensional networks approximately converges at large $\alpha$ to $\mathcal{S}_{\mathrm{QWS}}(\alpha)$ of the one-dimensional quarter-wave stack. A physical explanation of this universal behavior has to apply to both crystal and disordered networks even though the PBGs in these two cases have distinctly different physical properties. The crystal networks have anisotropic PBGs bounded above at one $k$ point and below by a different $k$ point along the band gap. The slope $\mathcal{S}(\alpha)$ of the single stop gaps does not agree as well with the one-dimensional quarter-wave stack formula as that of the three-dimensional complete PBG. In contrast, the PBGs of the disordered networks are isotropic, i.e., they have the same stop gap in every direction (apart from negligible statistical fluctuations) so that the upper and lower boundaries of the PBG are both set by any single $k$ point. Moreover, the eigenmodes above and below the PBGs of the disordered networks are localized - again in contrast to the crystal networks. Finally, a physical explanation has to explicate why it only applies if the networks are optimized according to the three stages of our procedure.

We believe the similar behaviors of $\mathcal{S}(\alpha), \mathcal{D}_{\mathbf{E}}(\alpha)$, and $\phi_{\text {opt }}(\alpha)$ provide important clues for understanding the universal curves of $\mathcal{S}(\alpha)$ reported here, but we have not yet found a solid theoretical explanation that ties these different observations together to explain the universal behaviors. The challenge is to identify how, from a combination of highly nonlinear physics and a diverse range of network geometries and topologies explored here, these behaviors emerge.

As a practical application, the discovery of this universal behavior at large $\alpha$ makes it possible to estimate for a given type of optimized heterostructure the photonic band gap-to-midgap ratio $\Delta(\alpha)$ for all $\alpha$ once one has determined it for a single $\alpha$ without any further extensive computations. On the more theoretical side, the fact that the behavior applies to optimized structures in three dimensions, whether crystalline or disordered, as well as to periodic layered media, indicates an unanticipated simplicity, despite the apparent nonlinear mathematics and complex physics involved in the PBG computation and the optimization procedure. A better understanding of this behavior will ultimately help to identify the relation between the structural features of optimized heterostructures and the formation of large PBGs, which may guide the design of improved photonic heterostructures.

We thank G. T. Barkema and N. Mousseau for pro- viding samples of CRNs and the Princeton Institute for Computational Science and Engineering (PICSciE) for the computational resources. This work was partially supported by the Princeton University Innovation Fund for New Ideas in the Natural Sciences. S. T. also gratefully acknowledges the support of the Air Force Office of Scientific Research Program on Mechanics of Multifunctional Materials and Microsystems under Grant No. FA9550-18-1-0514. M. A. K. also acknowledges funding by the Volkswagenstiftung via the ExperimentProjekt Mecke.

*mklatt@princeton.edu

$\dagger$ steinh@princeton.edu

‡ torquato@princeton.edu

[1] J. D. Joannopoulos, S. G. Johnson, J. N. Winn, and R. D. Meade, Photonic Crystals: Molding the Flow of Light, 2nd ed. (Princeton University Press, Princeton, 2008).

[2] M. Maldovan and E. L. Thomas, Nat. Mater. 3, 593 (2004).

[3] C. T. Chan, K. M. Ho, and C. M. Soukoulis, Europhys. Lett. 16, 563 (1991).

[4] W. Man, M. Megens, P. J. Steinhardt, and P. M. Chaikin, Nature 436, 993 (2005).

[5] M. C. Rechtsman, H.-C. Jeong, P. M. Chaikin, S. Torquato, and P. J. Steinhardt, Phys. Rev. Lett. 101, 073902 (2008).

[6] M. Florescu, S. Torquato, and P. J. Steinhardt, Proc. Natl. Acad. Sci. U.S.A. 106, 20658 (2009).

[7] K. Edagawa, S. Kanoko, and M. Notomi, Phys. Rev. Lett. 100, 013901 (2008).

[8] S. Imagawa, K. Edagawa, K. Morita, T. Niino, Y. Kagawa, and M. Notomi, Phys. Rev. B 82, 115116 (2010).

[9] S. F. Liew, J.-K. Yang, H. Noh, C. F. Schreck, E. R. Dufresne, C. S. O'Hern, and H. Cao, Phys. Rev. A 84, 063818 (2011).

[10] H. Yin, B. Dong, X. Liu, T. Zhan, L. Shi, J. Zi, and E. Yablonovitch, Proc. Natl. Acad. Sci. U.S.A. 109, 10798 (2012).

[11] K. Edagawa, Sci. Technol. Adv. Mater. 15, 034805 (2014).

[12] N. Muller, J. Haberko, C. Marichy, and F. Scheffold, Adv. Opt. Mater. 2, 115 (2014).

[13] S. Tsitrin, E. P. Williamson, T. Amoah, G. Nahal, H. L. Chan, M. Florescu, and W. Man, Sci. Rep. 5, 13301 (2015).

[14] S. Imagawa and K. Edagawa, Appl. Phys. A 123, 41 (2017).

[15] S. R. Sellers, W. Man, S. Sahba, and M. Florescu, Nat. Commun. 8, 14439 (2017).

[16] X. Li, A. Das, and D. Bi, Proc. Natl. Acad. Sci. U.S.A. 115, 6650 (2018).

[17] H. Men, K. Y. K. Lee, R. M. Freund, J. Peraire, and S. G. Johnson, Opt. Express 22, 22632 (2014).

[18] S. Torquato, Phys. Rep. 745, 1 (2018).

[19] M. Hejna, P. J. Steinhardt, and S. Torquato, Phys. Rev. B 87, 245204 (2013).

[20] V. L. Deringer, N. Bernstein, A. P. Bartók, M. J. Cliffe, R. N. Kerber, L. E. Marbella, C. P. Grey, S. R. Elliott, 
and G. Csányi, J. Phys. Chem. Lett. 9, 2879 (2018).

[21] M. A. Klatt, J. Lovrić, D. Chen, S. C. Kapfer, F. M. Schaller, P. W. A. Schönhöfer, B. S. Gardiner, A.-S. Smith, G. E. Schröder-Turk, and S. Torquato, Nat. Commun. 10, 811 (2019).

[22] S. Johnson and J. Joannopoulos, Opt. Express 8, 173 (2001).

[23] The overall CPU time utilized for this study was about 70 years on Intel Skylake CPUs.

[24] P. Yeh, Optical Waves in Layered Media (Wiley, Hoboken, NJ, 1988).

[25] H. A. Macleod, Thin-Film Optical Filters, 4th ed. (CRC Press, Boca Raton; London; New York, 2010).

[26] M. A. Klatt, P. J. Steinhardt, and S. Torquato, Proc. Natl. Acad. Sci. U.S.A. 116, 23480 (2019).

[27] S. T. Hyde, M. O'Keeffe, and D. M. Proserpio, Angew. Chem. Int. Ed. 47, 7996 (2008).

[28] B. D. Wilts, B. Apeleo Zubiri, M. A. Klatt, B. Butz, M. G. Fischer, S. T. Kelly, E. Spiecker, U. Steiner, and G. E. Schröder-Turk, Sci. Adv. 3, e1603119 (2017).

[29] T. Sunada, Not. Am. Math. Soc. 55, 8 (2008).

[30] H. S. Sözüer and J. W. Haus, J. Opt. Soc. Am. B 10, 296 (1993).
[31] M. Maldovan and E. L. Thomas, J. Opt. Soc. Am. B 22, 466 (2005).

[32] G. T. Barkema and N. Mousseau, Phys. Rev. B 62, 4985 (2000).

[33] S. Torquato and F. H. Stillinger, Phys. Rev. E 68, 041113 (2003).

[34] S. Torquato, Phys. Rev. E 82, 056109 (2010).

[35] S. Lloyd, IEEE Trans. Inf. Theory 28, 129 (1982).

[36] Y. Liu, W. Wang, B. Lévy, F. Sun, D.-M. Yan, L. Lu, and C. Yang, ACM Trans. Graph. 28, 101:1 (2009).

[37] Q. Du, M. Gunzburger, and L. Ju, Numer. Math. 3, 119 (2010).

[38] J. Zhang, M. Emelianenko, and Q. Du, Int. J. Numer. Anal. Model. 9, 950 (2012).

[39] C. Ruscher, J. Baschnagel, and J. Farago, Europhys. Lett. 112, 66003 (2015).

[40] C. Ruscher, S. Ciarella, C. Luo, L. M. C. Janssen, J. Farago, and J. Baschnagel, J. Phys. Condens. Matter 33, 064001 (2021).

[41] T. M. Hain, M. A. Klatt, and G. E. Schröder-Turk, J. Chem. Phys. 153, 234505 (2020).

[42] J. Haberko and F. Scheffold, Opt. Express 21, 1057 (2013). 


\title{
Supplemental Material: \\ Gap Sensitivity Reveals Universal Behaviors in Optimized Photonic Crystal and Disordered Networks
}

\author{
Michael A. Klatt, ${ }^{1,2}$ Paul J. Steinhardt, ${ }^{3}$ and Salvatore Torquato ${ }^{3,4}$ \\ ${ }^{1}$ Department of Physics, Princeton University, Princeton, New Jersey 08544, USA \\ ${ }^{2}$ Institut für Theoretische Physik, University of Erlangen-Nürnberg, Staudtstr. 7, 91058 Erlangen, Germany \\ ${ }^{3}$ Department of Physics, Princeton University, Princeton, NJ 08544, USA \\ ${ }^{4}$ Department of Chemistry, Princeton Institute for the Science and Technology of Materials, \\ and Program in Applied and Computational Mathematics, \\ Princeton University, Princeton, New Jersey 08544, USA
}

(Dated: July 16, 2021)

\section{S1. Simulation details}

Here we provide additional parameters for our network models and the computation of their (optimal) photonic band gaps (PBGs).

Network models

For the nearly hyperuniform network (NHN), we used the most annealed sample with 1000 vertices from Ref. [1]. For the network based on the molecular dynamics (MD) quench, we used the sample GAP-MD_quench_1e11_lastMDstep published together with Ref. [2]. The definition of our quantizer-based networks (QBNs) is based on the amorphous inherent structures of the quantizer energy from Ref. [3]; for references on the quantizer energy, see Refs. [3-10]. To construct our QBN, we start from a binomial point process (that is, a snapshot of the ideal gas in the canonical ensemble) and minimize the quantizer energy using the so-called Lloyd algorithm [11]: In each step, the Voronoi center of each cell is replaced by the center of mass of the cell. The algorithm converges to an amorphous inherent structure [3]. Here, we apply 10,000 steps of the Lloyd algorithm. The network is then constructed using the Delaunay tessellation as explained in the main text. Our definition of the QBNs allows the simulation of extended networks with a million vertices. For computational reasons, we here simulated a sample with 444 vertices.

The unit of length was chosen for each sample such that the number of vertices equals the volume of the sample. This choice corresponds to a unit number density $\rho=1$ for the vertices. The number of vertices per sample is 1000 for the NHN, 444 for the QBN, 512 for the MD quench, and 216 for the perturbed diamond networks. To check for system size effects, we also analyzed a continuous random network (CRN) by Barkema and Mousseau [12] with 216 vertices and a perturbed diamond network $(a=0.2)$ with 1000 vertices; see Fig. S4.

A single sample of a perturbed diamond or disordered network can have several complete PBGs between different bands. Typically, one of these PBGs is much larger than the others; see video S2 and Fig. S1. For each sample and each value of $\alpha$, we optimize the PBG for which we find the largest value of $\Delta(\alpha)$ (with few exceptions as explained below). The number of bands below this largest PBG is 1000 for the NHN, 509 for the MD quench, 444 for the QBN for $\alpha>9.3$ (for $\alpha \leq 9.3$, we used the same band number although the PBG at band number 442 is slightly larger), 1000 for the perturbed diamond network with 1000 vertices, 216 for the perturbed diamond networks with 216 vertices and with $a=0.1,0.2$, and 0.3 , and 215 for the perturbed diamond network with $a=0.4$. For the last sample, we found several small complete PBGs of roughly the same size so that the number of bands below the largest gap can differ for different values of $R$ and $\alpha$. The same applies to our perturbed CRN with $a=0.3$.

\section{Parameters}

The resolution $\mathcal{R}$, which is defined in the MIT Photonic Bands (MPB) software package as the number of voxels per unit length, was set to the following values during the optimization of the radii:

- $\mathcal{R}=16$ for the disordered networks and perturbed diamond networks,

- $\mathcal{R}=20$ for the diamond, hexagonal-diamond, and Laves networks, and 
- $\mathcal{R}=80$ for the simple cubic (SC) network.

For the final values of Figs. 2 and 3 in the main text, as well as for Fig. S4, we used the following resolutions:

- $\mathcal{R}=16$ for the perturbed diamond network with 1000 vertices,

- $\mathcal{R}=20$ for the disordered networks and perturbed diamond networks,

- $\mathcal{R}=80$ for the diamond, hexagonal-diamond, and Laves networks, and

- $\mathcal{R}=160$ for the simple cubic (SC) network.

The number of $k$ points for which we computed the eigenfrequencies are

- 10 for the NHN, MD quench, and the QBN,

- 22 for the perturbed diamond networks and the perturbed CRNs.

For the disordered networks, the $k$ points always include the high symmetry points (of the simple cubic simulation box). For the crystal networks, we computed the eigenfrequencies at 8 points between pairs of high symmetry points. The tolerance of the MPB eigensolver was $10^{-5}$.

The calculations at strong contrasts are more prone to voxelization errors because of the smaller values of the optimal radii. The estimation of the optimal radii is also more difficult at strong contrasts because the optimal gap size as a function of the radius $R$ becomes a skewed function (even close to the optimum). Finally, single defects in disordered or perturbed networks can strongly influence the optimal gap size. We, therefore, used the following improved parameters for some of the calculations at strong contrasts (to check their influence on the results, which was not found to be strong). To determine the optimal radii for the crystal networks, we used a resolution of 80 for the diamond network with $\alpha \geq 20$ and the laves network with $\alpha \geq 18$. Moreover, we used a resolution of 20 for the CRN with $\alpha=8.3,11.3$, and 13.0 .

Finally, in our study of system size effects in Fig. S4, the computation for the perturbed diamond network with 1000 vertices was computationally particularly expensive because of the large number of $k$-points that have to be considered (because the perturbed diamond network has an anisotropic band structure -in contrast to the NHN). Therefore, we did not optimize the radii separately but used the optimal radii of the perturbed diamond network with 216 vertices. We checked for $\alpha=4.8$ and 13 that these radii are too a good approximation also optimal for the network with 1000 vertices. We also checked that the resolution $\mathcal{R}=16$ was sufficient for our calculations by comparing the results for two stop gaps with the corresponding results for a resolution $\mathcal{R}=20$. The differences in the gap-to-midgap ratios were about $0.1 \%$, that is, within the accuracy of our study.

Volume fractions

We estimate the volume fractions $\phi$ of our networks using the dielectric filling fraction implemented in MPB. The MPB software smooths the discontinuous dielectric function at the resolution of the grid (to avoid convergence problems caused by the discretization) [13]. The dielectric filling fraction is then defined as:

$$
\varphi:=\frac{\langle\varepsilon\rangle-\min \varepsilon}{\max \varepsilon-\min \varepsilon},
$$

where $\langle\varepsilon\rangle, \min \varepsilon$, and $\max \varepsilon$ are the average, minimal, and maximal dielectric constant over all voxels. For a two-phase medium that consists of a high and a low dielectric material, the filling fraction $\varphi$ is equivalent to the volume fraction $\phi$ of the high dielectric phase.

[1] M. Hejna, P. J. Steinhardt, and S. Torquato, Phys. Rev. B 87, 245204 (2013).

[2] V. L. Deringer, N. Bernstein, A. P. Bartók, M. J. Cliffe, R. N. Kerber, L. E. Marbella, C. P. Grey, S. R. Elliott, and G. Csányi, J. Phys. Chem. Lett. 9, 2879 (2018).

[3] M. A. Klatt, J. Lovrić, D. Chen, S. C. Kapfer, F. M. Schaller, P. W. A. Schönhöfer, B. S. Gardiner, A.-S. Smith, G. E. Schröder-Turk, and S. Torquato, Nat. Commun. 10, 811 (2019).

[4] Y. Liu, W. Wang, B. Lévy, F. Sun, D.-M. Yan, L. Lu, and C. Yang, ACM Trans. Graph. 28, 101:1 (2009). 
[5] Q. Du, M. Gunzburger, and L. Ju, Numer. Math. 3, 119 (2010).

[6] S. Torquato, Phys. Rev. E 82, 056109 (2010).

[7] J. Zhang, M. Emelianenko, and Q. Du, Int. J. Numer. Anal. Model. 9, 950 (2012).

[8] C. Ruscher, J. Baschnagel, and J. Farago, Europhys. Lett. 112, 66003 (2015).

[9] C. Ruscher, S. Ciarella, C. Luo, L. M. C. Janssen, J. Farago, and J. Baschnagel, J. Phys. Condens. Matter 33, 064001 (2021).

[10] T. M. Hain, M. A. Klatt, and G. E. Schröder-Turk, J. Chem. Phys. 153, 234505 (2020).

[11] S. Lloyd, IEEE Trans. Inf. Theory 28, 129 (1982).

[12] G. T. Barkema and N. Mousseau, Phys. Rev. B 62, 4985 (2000).

[13] S. Johnson and J. Joannopoulos, Opt. Express 8, 173 (2001). 
S2. Photonic band structures
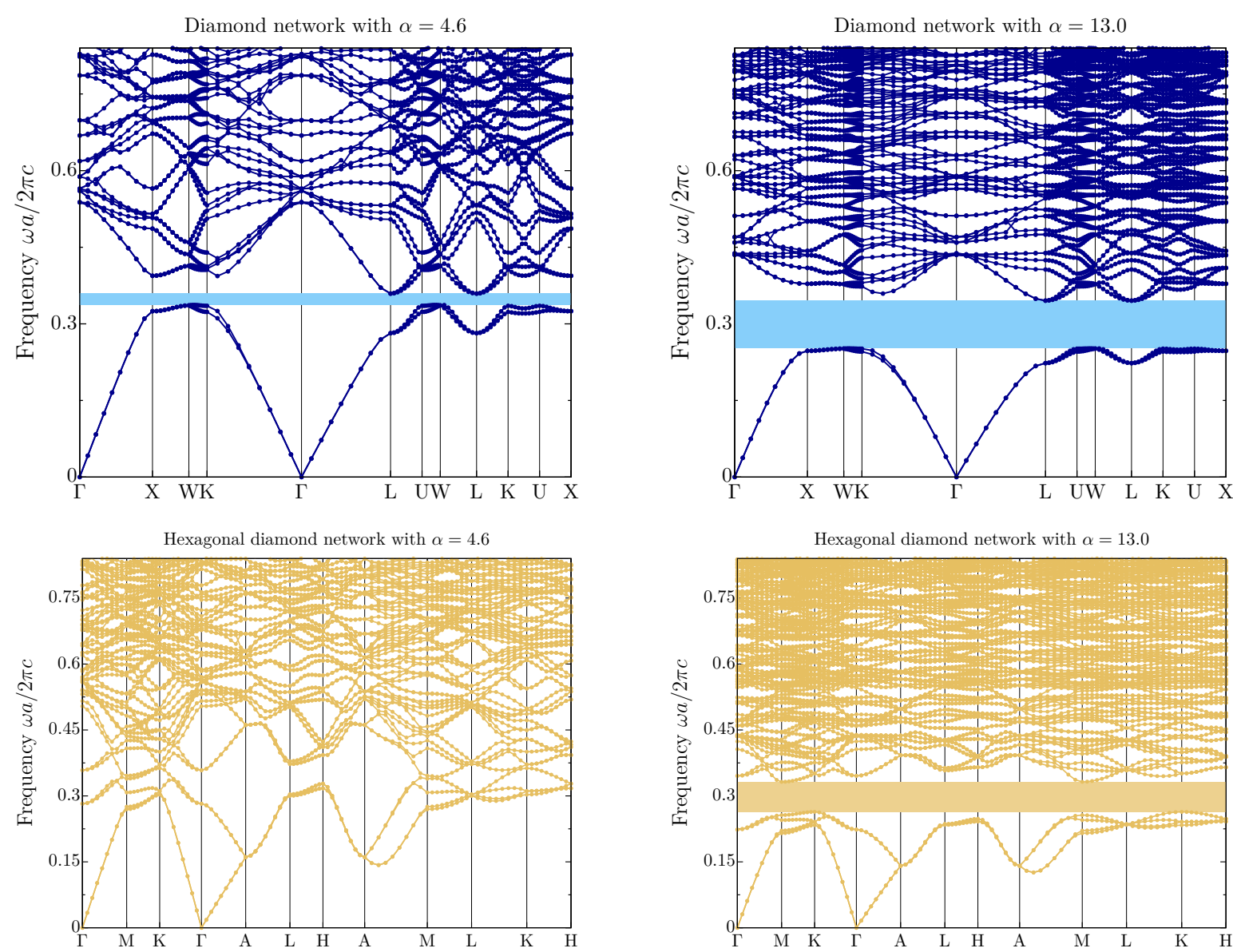

Perturbed dia. network $(a=0.2)$ with $\alpha=4.6$
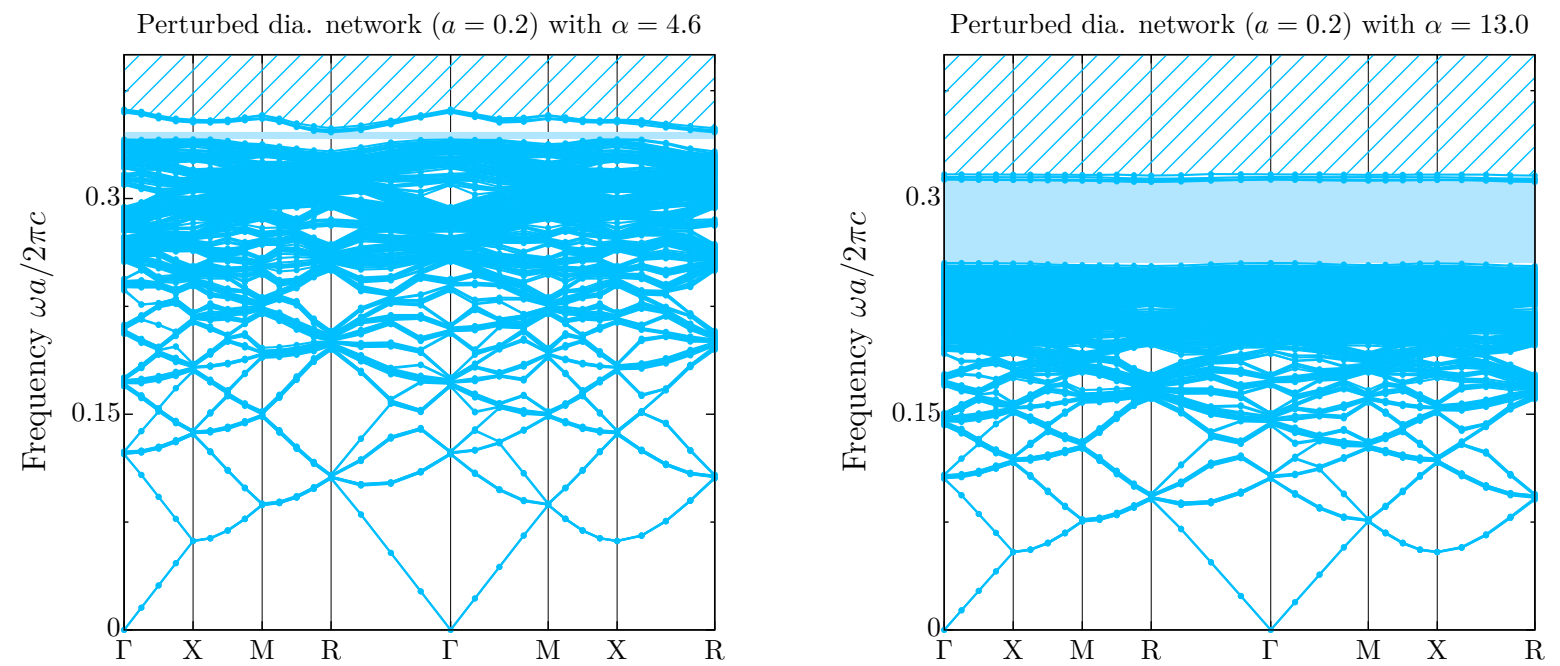

FIG. S1. Photonic band structures for some of the (anisotropic) crystal and perturbed crystal networks at different dielectric contrasts $\alpha$; see also video S2. 

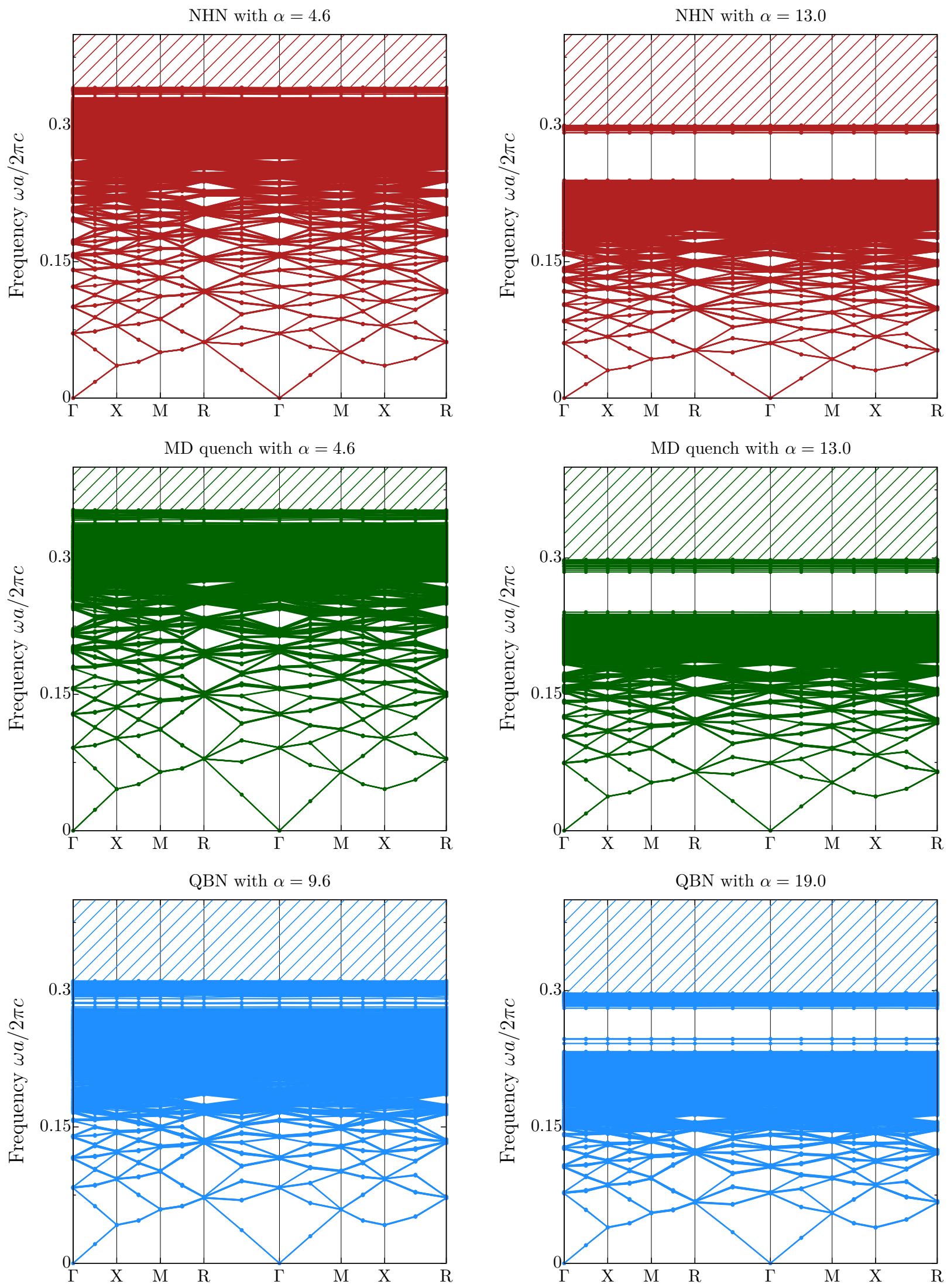

FIG. S1. (Continued) Photonic band structures of (isotropic) disordered networks at different dielectric contrasts $\alpha$; see also video $\mathrm{S} 2$. 
S3. Derivative of the optimal volume fraction

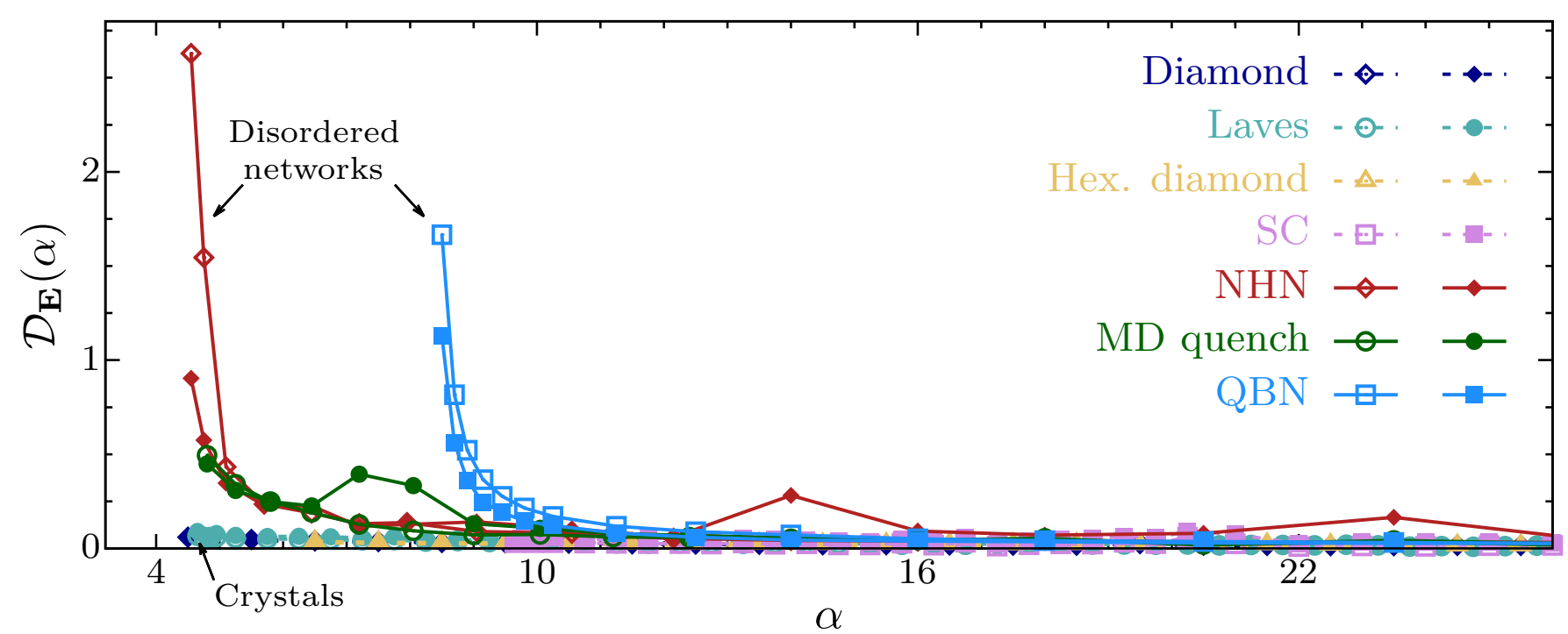

FIG. S2. The average change of the electric field intensity with the dielectric contrast $\alpha$ for the bands below (open symbols) and above (solid symbols) the PBG. At low $\alpha$, the configuration of the electric field changes more strongly for the disordered networks than for the photonic crystals.

S4. Derivative of the optimal volume fraction

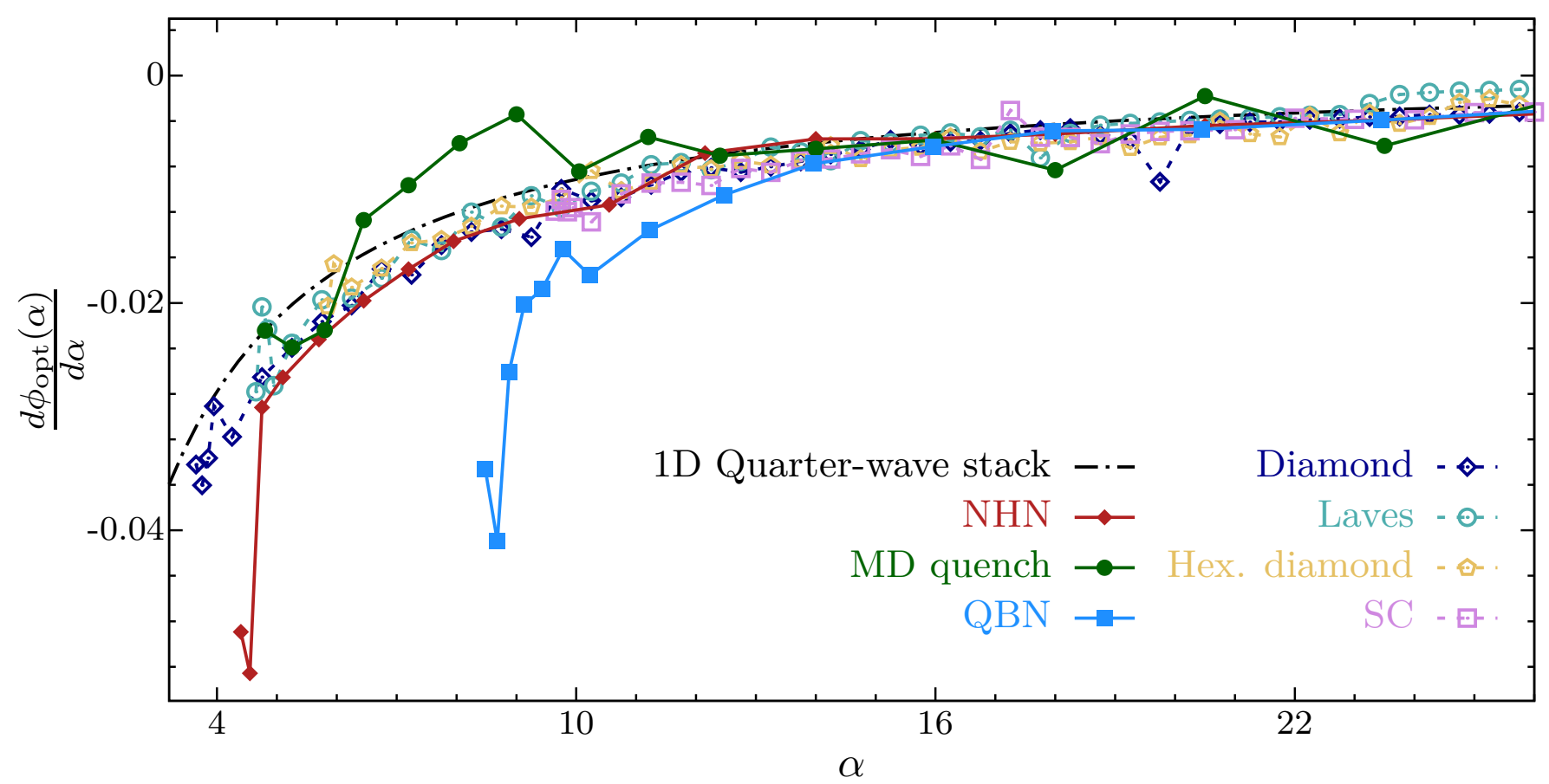

FIG. S3. The derivative of the optimal volume fraction, $d \phi_{\text {opt }}(\alpha) / d \alpha$ for our crystal and disordered networks approximately agrees at large $\alpha$ with the analytic result for the $1 \mathrm{D}$ quarter-wave stack, $d \phi_{\mathrm{QWS}}(\alpha) / d \alpha=-1 /\left(2 \alpha^{3 / 2}+4 \alpha+2 \sqrt{\alpha}\right)$, shown by the dashed-dotted line. 
S5. System size effects
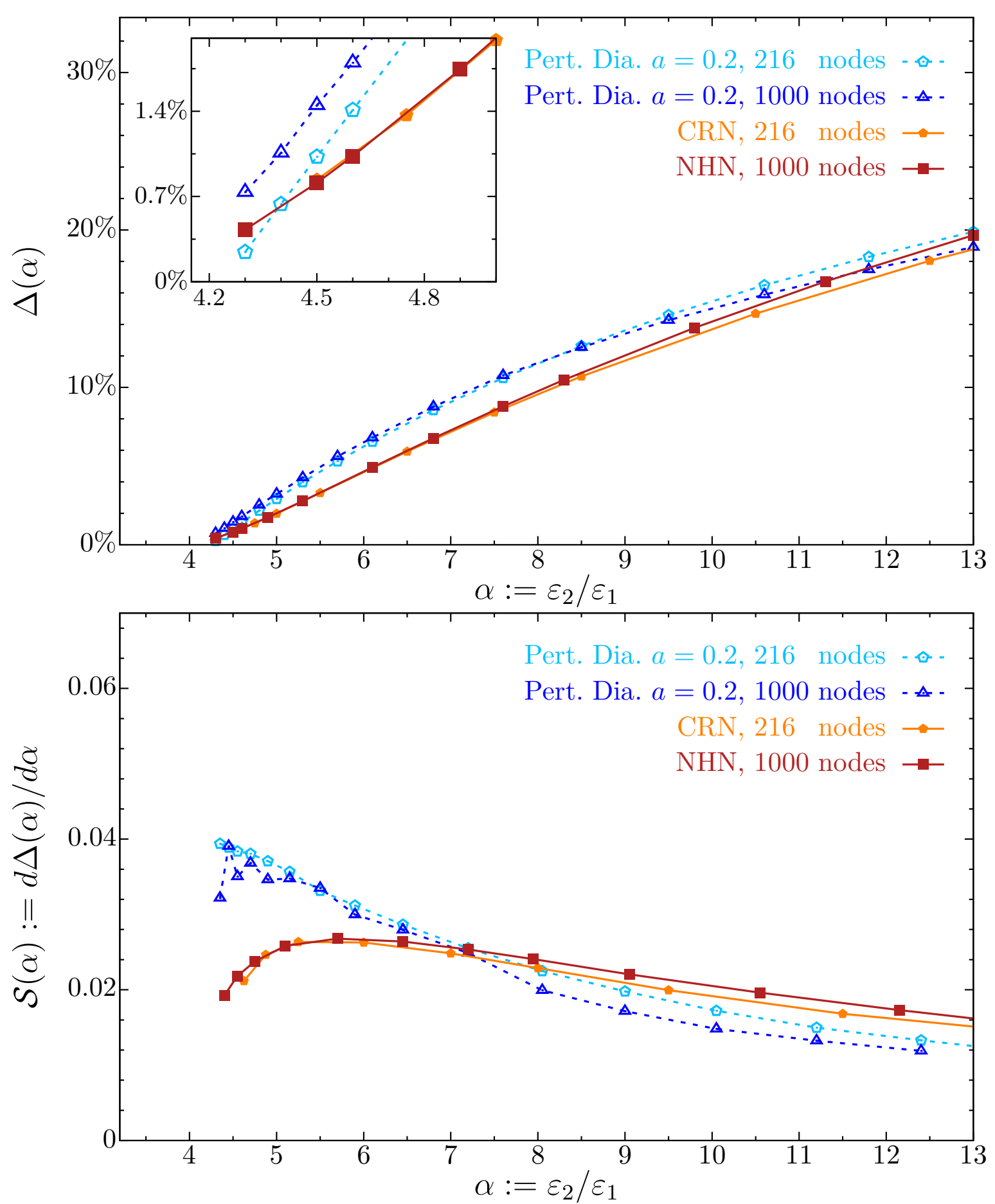

FIG. S4. The gap plot (top) and gap-sensitivity plot (bottom) for the NHN (1000 vertices) and the perturbed diamond network with $a=0.2$ (216 vertices) are compared to similar networks at different system sizes: a CRN with 216 vertices and a perturbed diamond network with 1000 vertices, respectively. The curves at different system sizes agree within the systematic errors and statistical fluctuations that can be expected between different samples; that is, no strong system size effect is observed. 